\title{
GIMAP7 as a Potential Predictive Marker for Pan-Cancer Prognosis and Immunotherapy Efficacy
}

\author{
Yan Qin (1D ${ }^{1, *}$, He Liu' ${ }^{1} *$, Xiaoliang Huang ${ }^{2}$, Lihaoyun Huang ${ }^{2}$, Lixian Liao ${ }^{2}$, Jiasheng Li $^{2}$, Lihua Zhang ${ }^{2}$, \\ Wei Li', Jianrong Yang' \\ 'Department of Health Management, The People's Hospital of Guangxi Zhuang Autonomous Region \& Research Center of Health Management, \\ Guangxi Academy of Medical Sciences, Nanning, Guangxi, 53002I, People's Republic of China; ${ }^{2}$ Guangxi Medical University Cancer Hospital, Nanning, \\ Guangxi, 53002I, People's Republic of China \\ *These authors contributed equally to this work
}

Correspondence: Jianrong Yang; Wei Li, Health Examination Center, The People's Hospital of Guangxi Zhuang Autonomous Region, Email gandansurgery20I4@I63.com; Iw6666gx@aliyun.com

Background: GIMAP, a GTP enzyme of immune-related proteins, plays a crucial role in immune mechanisms. Investigating GIMAP7 expression in pan-cancer can provide efficient guidance for predicting clinical prognosis and identifying novel immunotherapy targets.

Methods: Gene expression in different tumour types and stages was analysed based on The Cancer Genome Atlas and the GenotypeTissue Expression database. An immunohistochemical assay was used to explore the differences in GIMAP7 protein levels in different tumour types and stages. Further, the cBioPortal was used to obtain the genetic variation characteristics of GIMAP7. Kaplan-Meier analysis and multivariable Cox regression analysis were performed to assess the prognostic value of GIMAP7. The pathways affected by GIMAP7 were studied based on gene set enrichment analysis, and the correlation between GIMAP7 expression and immune infiltration was determined using the TIMER2 database and the CIBERSORT method. ESTIMATE was used to analyse the correlation between GIMAP7 expression and ESTIMATE, immune and stromal scores. In addition, the correlation between GIMAP7 and immunoregulators was analysed. Furthermore, tumour mutational burden and microsatellite instability were evaluated using Spearman correlation assay.

Results: GIMAP7 expression was significantly low and predicted better survival status in most tumour types. GIMAP7 was positively correlated with the abundance of CD8 + and CD4 $+\mathrm{T}$ cells in the tumour microenvironment. However, the high expression of GIMAP7 was negatively correlated with tumour mutations in uveal melanoma and colon adenocarcinoma. A correlation between GIMAP7 and microsatellite instability was found in rectal adenocarcinoma. Additionally, GIMAP7 presented a robust correlation between immune modulators and immunotherapeutic markers. Moreover, high GIMAP7 expression was significantly related to immune-relevant pathways.

Conclusion: This study suggests the potential role of GIMAP7 as a prognostic and immunotherapeutic marker in pan-cancer, laying the groundwork for prospective functional and mechanistic experiments and their impact in the clinical setting.

Keywords: immune-associated nucleotide-binding protein 7, pan-cancer analysis, immunoregulation, immunotherapy, CD8-positive $\mathrm{T}$ cells

\section{Introduction}

Cancer is expected to rank as the leading cause of death globally and is considered to be the single most important barrier to increasing life expectancy in the 21 st century. ${ }^{1}$ Tumours possess a complex microenvironment composed of tumour malignant cells, ${ }^{2}$ which makes tumour therapy difficult to implement. Given the prevalence of tumours and the complex process of tumorigenesis, it is crucial to explore in-depth the expression of relevant genes in pan-cancer and to assess their levels for clinical treatment and prognostic prediction. 
GIMAP has been identified as a GTPase of immune-associated proteins that play a crucial role in immune mechanisms, especially $\mathrm{T}$ cell production, development and stable immune processes. ${ }^{3}$ GIMAP mRNAs are majorly expressed in the spleen and lymphoid, with GIMAP7 predominantly found in the endoplasmic reticulum and Golgi apparatus of lymphocytes. ${ }^{4}$ The AGI1 domain has been described as a GTPase involved in binding nucleotide with a helical segment to moderate interaction with fellow proteins, both of which are necessary components of GIMAPs. ${ }^{5}$ Studies on the association of GIMAPs with individual cancers and diseases have identified the GIMAP family as a potential diagnostic and prognostic biomarker of lung adenocarcinoma. ${ }^{6}$ Additionally, the dysregulation of GIMAP5 and GIMAP6 has been reported in hepatocellular carcinoma. GIMAP family is also closely related to tumour immunity. GIMAP1 is essential for the survival of naive and activated B cells, ${ }^{7}$ while GIMAP3 and GIMAP5 play essential roles in haematopoiesis and $\mathrm{T}$ cell homeostasis through their ability to affect the activity of Bcl-2 family members. GIMAP4 accelerates T-cell death. ${ }^{8}$ However, studies on GIMAP7, a member of the GIMAP family that has a specific structure and regulates other GIMAP family members, are scarce. ${ }^{9}$

This study comprehensively analyzes the expression of GIMAP7 in pan-cancer, including differential gene expression analysis, protein correlation analysis, pathway analysis, prognostic analysis of different tumour types and stages, and compares the correlation of GIMAP7 expression and immune infiltrating cells and immunoregulatory factors. The results indicate that GIMAP7 could be a competent prognostic biomarker and closely related to the immune mechanism, which illustrates its potential as an immunotherapeutic agent for various cancers.

\section{Methods}

\section{Clinical Specimens}

Paired cancers and paracancerous tissues were derived from 20 patients with breast cancer and 20 patients with bladder cancer. All patients were diagnosed with breast or bladder cancer and had not received chemotherapy or radiotherapy before tissue collection. Written informed consent was acquired from all patients. The study was approved by the Ethics and Anthropology Committee and performed following relevant guidelines and regulations.

\section{Data Collection}

RNA expression and clinical data were downloaded from The Cancer Genome Atlas (TCGA) and Pan-Cancer Atlas Hub of UCSC Xena database, ${ }^{10}$ which were batch-corrected to adjust for platform differences between the GAII and HiSeq Illumina sequencers as previously described. ${ }^{11}$ Additionally, Genotype-Tissue Expression (GTEx) data were acquired from the Xena database, whereas DNA copy numbers were downloaded from the cBioPortal database (https://www. cbioportal.org/). To verify the predictive value of GIMAP7 in immunotherapy response, gene expression data and clinical data of IMvigor210, ${ }^{12}$ which is an immunotherapy cohort of uroepithelial carcinoma, were downloaded from the Gene Expression Omnibus (GEO) database.

\section{Immunohistochemical Staining}

All specimens were immersed in formalin. Before staining, tissues were cut to $5 \mu \mathrm{m}$ thickness and placed on glass slides. Endogenous peroxidase activity was inhibited and blocked by de-paraffinising and rehydrating using $5 \%$ bovine serum albumin at $37^{\circ} \mathrm{C}$ for $30 \mathrm{~min}$. The treated sections were incubated with anti-GIMAP7 at $4^{\circ} \mathrm{C}$ overnight and washed thrice with phosphate-buffered saline (PBS). Following this, the sections were incubated with a secondary antibody at $37^{\circ} \mathrm{C}$ for $30 \mathrm{~min}$. After further three washes with PBS, the sections were developed in diaminobenzidine. Microscopic images were obtained using light microscopy.

\section{Protein Level Analysis}

The Human Protein Atlas (HPA: https://www.proteinatlas.org) database was used to study the protein levels of GIMAP7 in human tumours and normal tissues. The String (https://string-db.org/) database was used to construct a protein-protein interaction network (PPI) for GIMAP7. 


\section{GIMAP7 Differential Expression Analysis}

Differences in GIMAP7 mRNA expression levels between tumour and normal tissues for different cancer types were determined using the SangerBox software. ${ }^{13}$ A comparison of cancer and paracancerous samples in the TCGA database was performed, and considering the small number of paracancerous tissues in the TCGA database, the corresponding normal samples from the GTEx database were added for secondary comparison. $t$-test was used to evaluate the differences in GIMAP7 expression between cancer and non-cancer tissues.

\section{Genetic Alteration Analysis}

Genetic variant characterisation of GIMAP7 was determined using the cBioPortal (https://www.cbioportal.org/) website via the "Quick Selection" and "TCGA Pan-Cancer Atlas Study" options. Moreover, the mutation frequency, copy number variation and mutation type were queried from the "Cancer Type Summary" module of the TCGA database.

\section{Survival Analysis}

Kaplan-Meier analysis was used to analyse the survival difference between the high- and low-expression groups to evaluate the prognostic value of GIMAP7. Multivariable Cox regression analysis was performed using the TCGA database to study the relationship between GIMAP7 expression and overall survival (OS), progression-free survival interval (PFI) and disease-specific survival (DSS) after adjusting for age and tumour stage. P-values and hazard ratio (HR) with $95 \%$ confidence intervals (CI) were determined for each cancer type.

\section{Gene Set Enrichment Analysis (GSEA)}

The pathways affected by GIMAP7 were investigated using GSEA via the cluster Profiler R package; the fold change in the average expression of genes between patients with high and low GIMAP7 expression was used to gain a list of classified genes that represented the input file. The entire biological process was evaluated according to the Kyoto Encyclopedia of Genes and Genomes (KEGG) and HALLMARK pathways.

\section{Immune Infiltrate Analysis}

The association of GIMAP7 expression with immune infiltration was acquired using the TIMER2 database and CIBERSORT method. The correlation between GIMAP7 expression and cellular abundances, such as CD4 $+\mathrm{T}$ cells, CD8 + T cells, neutrophils, macrophages, eosinophils and natural killer cells, was calculated using Pearson's correlation coefficient. The correlation between GIMAP7 expression and ESTIMATE score, immune score and the stromal score was determined using ESTIMATE.

\section{Immune Checkpoint Blockade (ICB) Therapy Response Prediction}

Potential ICB response was predicted using the Tumour Immune Dysfunction and Exclusion (TIDE) algorithm. ${ }^{14}$ TIDE applies a set of gene expression markers to estimate two distinct mechanisms of tumour immune evasion, including the dysfunction of tumour infiltration cytotoxic T lymphocytes (CTL) and exclusion of CTL by immunosuppressive factors. Patients with high TIDE scores exhibit a higher chance of tumour immune escape and thereby exhibit a lower response rate to ICB treatment.

\section{Statistical Analyses}

Transcripts per kilobase of exon model per million mapped reads serve as the unit of mRNA expression. The statistically significant differences between non-normally distributed variables were analysed using the Mann-Whitney $U$-test and normally distributed variables were reckoned by adopting the unpaired Student's $t$-test. To compare more than two groups, Kruskal-Wallis was used as a non-parametric method and adopted the one-way ANOVA test as a parametric method. Spearman correlation was used to test the correlation between GIMAP7 mRNA expression and tumour mutational burden (TMB), microsatellite instability (MSI) and TIDE score. The R software (version 3.5.0) was used 
to conduct all statistical analyses, and all statistical $\mathrm{P}$ values were two-sided, with $\mathrm{P}<0.05$ denoting statistical significance.

\section{Results}

\section{Analysis of GIMAP7 Expression in Pan-Cancers}

The evaluation of GIMAP7mRNA expression in pan-cancers via the Oncomine database showed that GIMAP7 had significantly low expression in most tumours, such as bladder cancer, breast cancer, colorectal cancer, stomach cancer, head and neck cancer, lung cancer, kidney cancer, leukaemia, lymphoma and sarcoma. Contrastingly, it was highly expressed in brain and central nervous system cancer and oesophageal cancer (Figure 1A). Additionally, analysis of the differential expression of GIMAP7 based on cancer and paracancerous tissue samples from the TCGA database showed that GIMAP7 had significantly lower expression in cancer tissues than paraneoplastic tissues, including bladder urothelial carcinoma (BLCA), breast invasive carcinoma (BRCA), colon adenocarcinoma (COAD), kidney renal papillary cell carcinoma (KIRP), lung adenocarcinoma (LUAD), lung squamous cell carcinoma (LUSC), rectum adenocarcinoma (READ), uterine corpus endometrial carcinoma (UCEC), thyroid carcinoma (THCA) and kidney chromophobe (KICH)
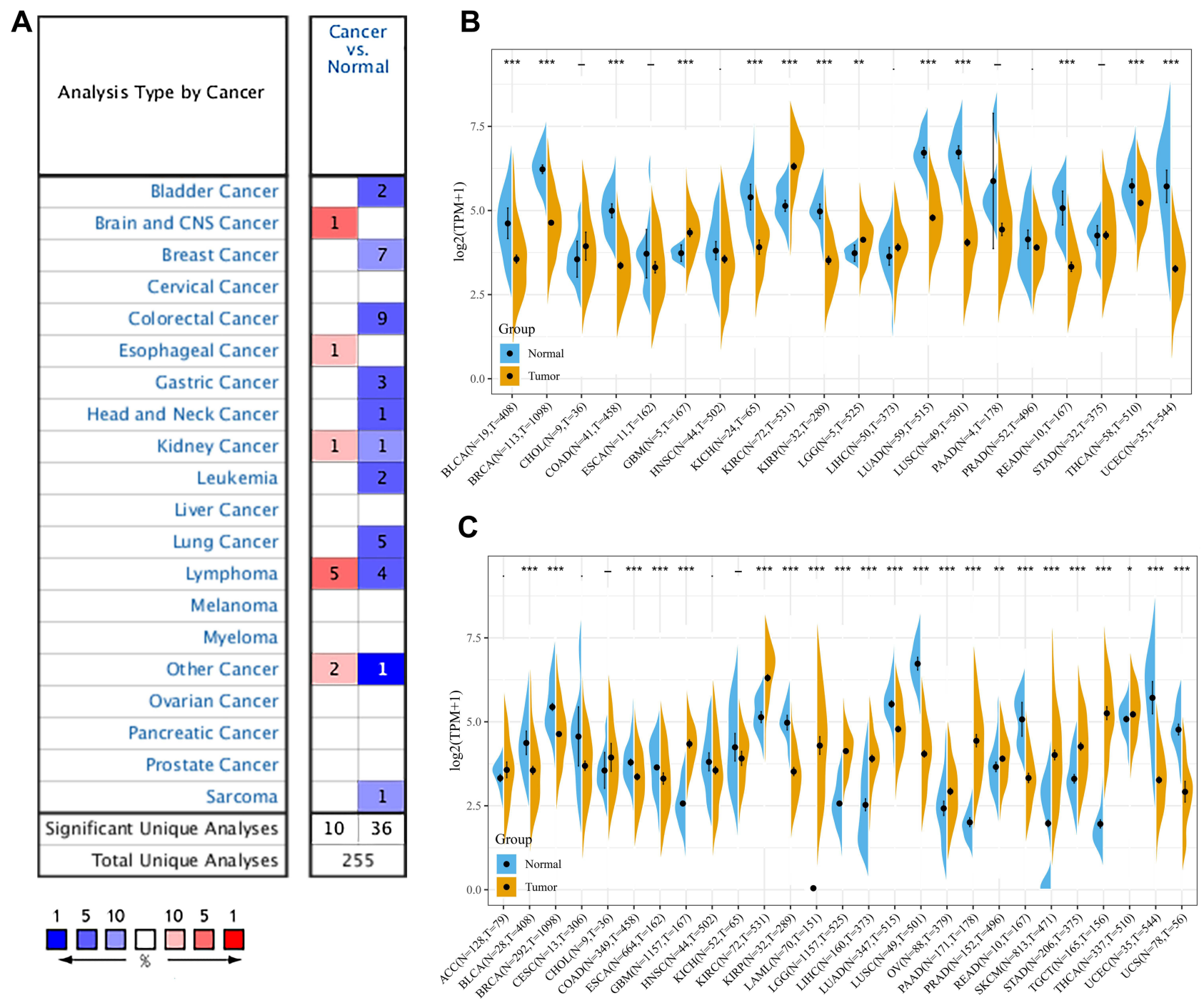

Figure I Pan-cancer GIMAP7 expression analysis. (A) The expression of GIMAP7 in different cancers and normal tissues in the Oncomine database. The number in each cell indicates the number of datasets. (B) The expression of GIMAP7 in tumours and adjacent normal tissues in the pan-cancer data of The Cancer Genome Atlas (TCGA) cohorts. (C) GIMAP7 expression in tumour tissues from TCGA cohorts and normal tissues from TCGA and Genotype-Tissue Expression cohorts. *P<0.05, **P $<0.01$, ***P $<0.00$ I. 
$(\mathrm{P}<0.001)$. However, its expression was significantly high in glioblastoma multiforme (GBM, $\mathrm{P}<0.001)$, brain lowergrade glioma (LGG, $\mathrm{P}<0.01$ ) and kidney renal clear cell carcinoma (KIRC, $\mathrm{P}<0.001$, Figure 1B). Due to the small number of normal samples in TCGA, the normal tissue data from the GTEx database were integrated with the TCGA tumour tissue database and the expression differences were analysed in 27 tumour types. GIMAP7 had low expression in 11 tumour types. Moreover, GIMAP7 in ovarian serous cystadenocarcinoma (OV), GBM, testicular germ cell tumours (TGCT), acute myeloid leukaemia (LAML), pancreatic adenocarcinoma (PAAD), liver hepatocellular carcinoma (LIHC), stomach adenocarcinoma (STAD) and skin cutaneous melanoma (SKCM) $(\mathrm{P}<0.001)$ was highly expressed (Figure 1C).

\section{Analysis of the Relationship Between GIMAP7 Expression and Tumour Stage}

The expression of GIMAP7 was evaluated in different cancer stages, which was defined according to the World Health Organization, and the expression of GIMAP7 was found to be significantly lower in the advanced stages of head and neck squamous cell carcinoma (HNSC, $\mathrm{P}=0.011)$, KIRC $(\mathrm{P}=0.0024)$, TGCT $(\mathrm{P}=0.0014)$ and THCA $(\mathrm{P}=1.7 \mathrm{e}-07)$ (Figure 2A-D). GIMAP7 expression decreased in patients with adrenocortical carcinoma (ACC), cholangiocarcinoma (CHOL), KICH and KIRP at tumours stage III-IV, but the difference was not statistically significant (Supplementary Figure 1).

\section{Genetic Alteration Analysis of GIMAP7}

The gene variants of GIMAP7 were investigated for different tumours in the TCGA cohort. The results showed that GIMAP7 had the highest frequency of mutations $(>8 \%)$ in patients with SKCM, followed by OV (Supplementary Figure 2A). Based on the type and location data of GIMAP7 gene variants, GIMAP7-associated missense mutations were the major type of gene variants, accounting for 95 loci. The remaining variants accounted for 19 truncating mutations and one splicing mutation (Supplementary Figure 2B). Additionally, copy number variation was negatively correlated with SKCM, KIRC and KIRP (Supplementary Figure 2C).

\section{Analysis of GIMAP7 Protein Levels}

The protein levels of GIMAP7 in different tumours were explored using the HPA database, wherein the level of GIMAP7 in bladder, lung and colorectal cancers was decreasing (Figure 3A-F). Further, immunohistochemistry and statistical analysis were performed on the patients with paired breast cancer and bladder cancer, which showed significantly decreased expression of GIMAP7 in breast cancer $(\mathrm{P}=0.0063)$ and bladder cancer $(\mathrm{P}=0.034)$. This result is consistent with previously reported data using the HPA database (Figure 4A-D). Furthermore, a PPI network was constructed that revealed GIMAP7's association with GIMAP1, GIMAP4, GIMAP5, GIMAP6, GIMAP8, ADCY4, SDR39U1, CD8A, GZMA and TMEM102 proteins (Supplementary Figure 3).
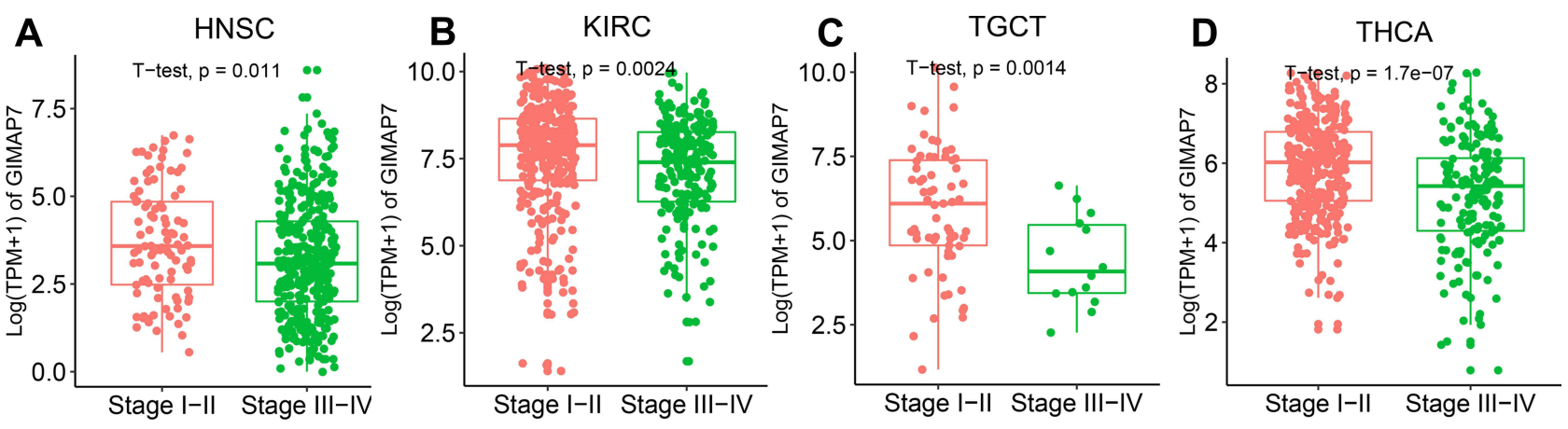

Figure 2 Pan-cancer GIMAP7 expression in different cancer stages as defined by World Health Organization. (A-D) Comparison of GIMAP7 expression in the early and advanced stages of different tumours. 

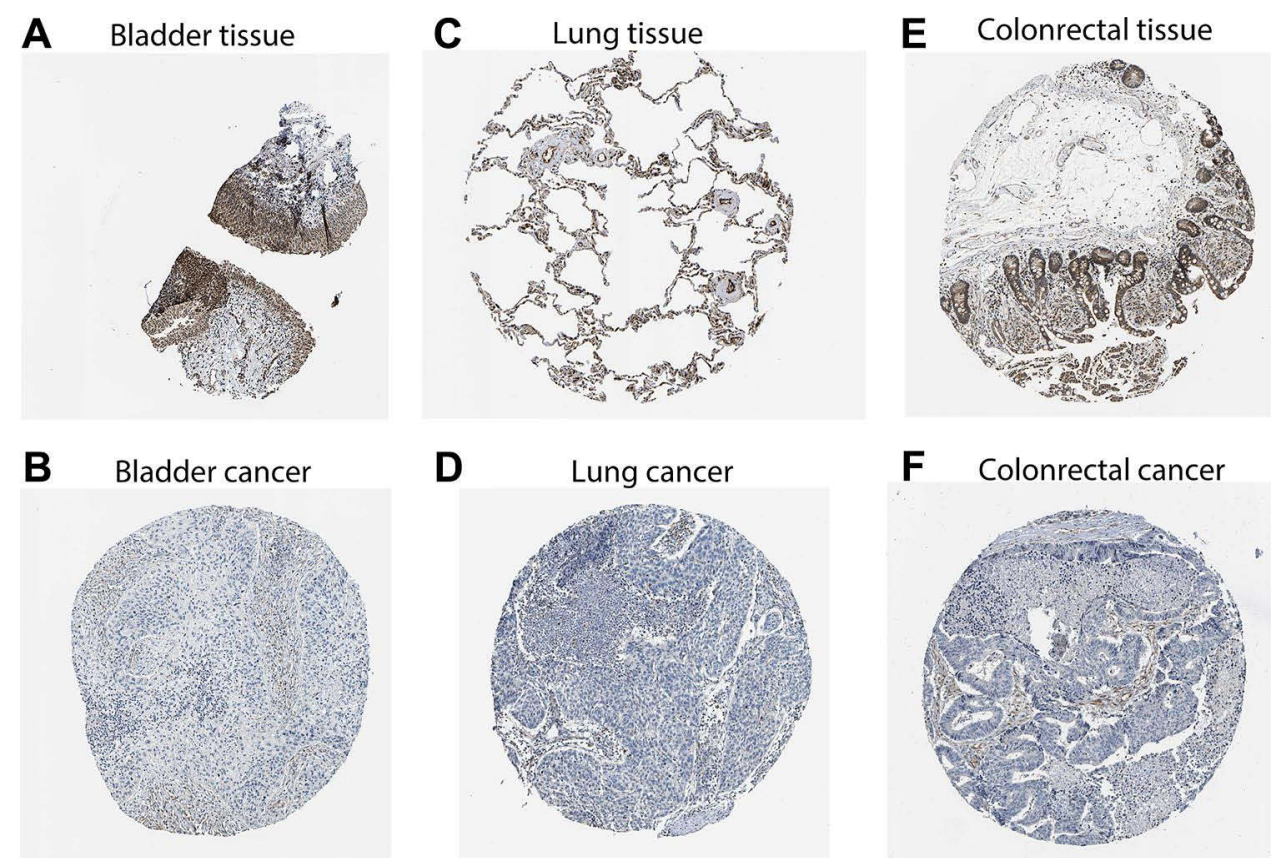

Figure 3 Protein levels of GIMAP7 in human tumours and normal tissues from the Human Protein Atlas database. (A, C, E) Normal bladder, lung and colorectum; (B, D, F) bladder cancer, lung cancer and colorectal cancer, respectively.

\section{Prognostic Analysis of GIMAP7 Using the TCGA Database}

The potential prognostic value of GIMAP7 was determined using Kaplan-Meier analysis and multivariable Cox Regression. Kaplan-Meier analysis showed that the high expression of GIMAP7 was associated with better prognosis in most cancers (Supplementary Figure 4). Furthermore, GIMAP7 was found to be a positive protective factor in ACC (P $=0.048, \mathrm{HR}=0.47), \mathrm{BLCA}(\mathrm{P}=0.009, \mathrm{HR}=0.56)$, BRCA $(\mathrm{P}=0.005, \mathrm{HR}=0.64)$, endocervical adenocarcinoma (CESC, $\mathrm{P}=0.002$, HR $=0.48)$, HNSC $(\mathrm{P}=0.002$, HR $=0.64)$, LIHC $(\mathrm{P}=0.001, \mathrm{HR}=0.57)$, LUAD $(\mathrm{P}<0.001, \mathrm{HR}=$ $0.51)$, KIRC $(\mathrm{P}<0.001, \mathrm{HR}=0.45)$, OV $(\mathrm{P}<0.001, \mathrm{HR}=0.65)$, PAAD $(\mathrm{P}=0.015, \mathrm{HR}=0.61)$ and sarcoma (SARC) $(\mathrm{P}$ $<0.001, \mathrm{HR}=0.39)$ ( Supplementary Figure 4A). Regarding DSS, GIMAP7 was indicated to be a protective factor in ACC $(\mathrm{P}=0.027, \mathrm{HR}=0.43)$, BLCA $(\mathrm{P}=0.006, \mathrm{HR}=0.5)$, BRCA $(\mathrm{P}=0.016, \mathrm{HR}=0.59)$, CESC $(\mathrm{P}=0.003, \mathrm{HR}=$ 0.37), HNSC ( $\mathrm{P}<0.001$, HR $=0.45)$, LIHC $(\mathrm{P}=0.009, \mathrm{HR}=0.56)$, LUAD $(\mathrm{P}<0.001$, HR $=0.5), \mathrm{KIRC}(\mathrm{P}<0.001, \mathrm{HR}$ $=0.36), \mathrm{OV}(\mathrm{P}<0.001, \mathrm{HR}=0.61), \mathrm{PAAD}(\mathrm{P}=0.023, \mathrm{HR}=0.59)$ and THCA $(\mathrm{P}<0.001, \mathrm{HR}=0.11)($ Supplementary Figure 4B). Additionally, GIMAP7 is found to be a protective factor in terms of PFI in ACC $(\mathrm{P}=0.003, \mathrm{HR}=0.39)$, BRCA $(\mathrm{P}<0.001, \mathrm{HR}=0.51)$, BLCA $(\mathrm{P}<0.001, \mathrm{HR}=0.49)$, CESC $(\mathrm{P}=0.004, \mathrm{HR}=0.51), \mathrm{COAD}(\mathrm{P}=0.044, \mathrm{HR}=$ 0.64), CHOL ( $\mathrm{P}=0.018, \mathrm{HR}=0.34)$, HNSC $(\mathrm{P}<0.001$, HR $=0.46)$, KIRC $(\mathrm{P}<0.001$, HR $=0.45), \mathrm{LIHC}(\mathrm{P}<0.001$, $\mathrm{HR}=0.48$ ), pheochromocytoma and paraganglioma ( $\mathrm{PCPG}, \mathrm{P}=0.005, \mathrm{HR}=0.24), \mathrm{OV}(\mathrm{P}=0.004, \mathrm{HR}=0.71)$, PAAD $(\mathrm{P}=0.005, \mathrm{HR}=0.58)$, THCA $(\mathrm{P}<0.001, \mathrm{HR}=0.28)$, UCEC $(\mathrm{P}=0.003, \mathrm{HR}=0.38), \mathrm{SARC}(\mathrm{P}=0.002, \mathrm{HR}=0.6)$, LUAD $(\mathrm{P}=0.002, \mathrm{HR}=0.64)$, mesothelioma $(\mathrm{P}=0.03, \mathrm{HR}=0.48)$ and TGCT $(\mathrm{P}=0.009, \mathrm{HR}=0.3)$ ( $\underline{\text { Supplementary }}$ Figure $4 \mathrm{C})$. As the patient's age and tumour stage are important factors that affect prognosis, multivariate Cox regression model was used to assess the prognostic value of GIMAP7 after adjusting for the patient's age and tumour stage. The result of OS revealed that GIMAP7 was significantly related to better prognosis of BLCA ( $\mathrm{P}=0.03, \mathrm{HR}=0.60)$, BRCA $(\mathrm{P}=0.01, \mathrm{HR}=0.60), \operatorname{CESC}(\mathrm{P}=0, \mathrm{HR}=0.49), \operatorname{HNSC}(\mathrm{P}=0, \mathrm{HR}=0.63), \mathrm{KIRC}(\mathrm{P}=0, \mathrm{HR}=0.48), \mathrm{LIHC}(\mathrm{P}=0, \mathrm{HR}$ $=0.58), \mathrm{LUAD}(\mathrm{P}=0, \mathrm{HR}=0.51), \mathrm{OV}(\mathrm{P}=0, \mathrm{HR}=0.65), \mathrm{SARC}(\mathrm{P}=0, \mathrm{HR}=0.38)$ and $\mathrm{PAAD}(\mathrm{P}=0.01, \mathrm{HR}=0.59)$ (Figure 5A). Similar to OS, DSS results indicated that the high expression of GIMAP7 could be significantly related to a better prognosis in 15 out of 32 tumours (Figure 5B). The results for PFI indicate that high expression of GIMAP7 is significantly associated with better PFI in 20 out of 32 tumours (Figure 5C). These results suggest that GIMAP7 could be an independent prognostic marker for various tumours. 

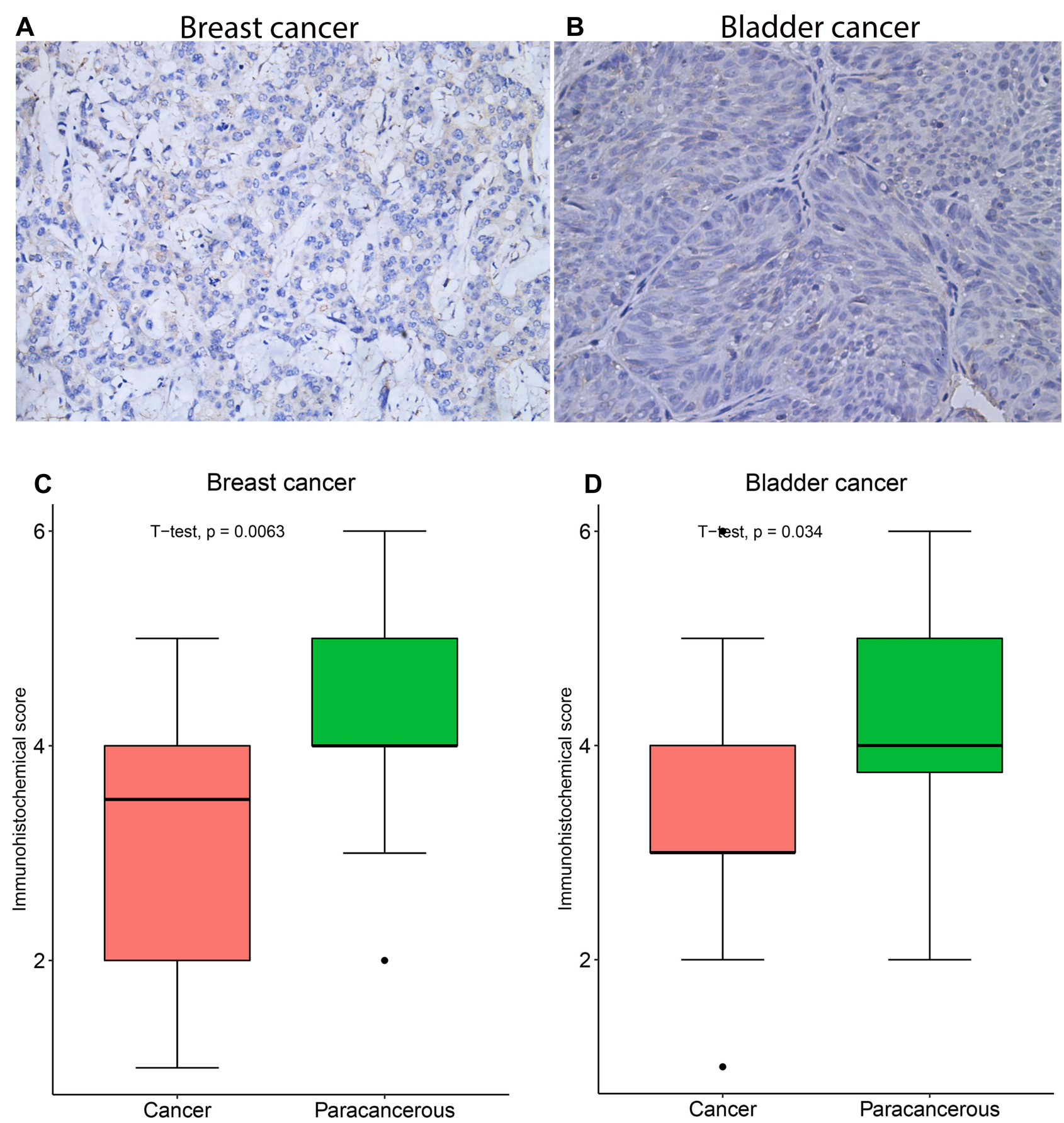

Figure 4 Immunohistochemical staining results and statistical graphs of GIMAP7 protein in breast and bladder cancers. (A and B) The 20x immunohistochemical images of breast cancer and bladder cancer, respectively. (C and D) The statistical results of 20 pairs of breast cancer and bladder cancer tissues, respectively.

\section{GSEA of GIMAP7 in KEGG and HALLMARK Pathways}

GSEA was used to identify the pathways associated with GIMAP7. According to KEGG, pathways related to cytokine receptor interactions, chemokine signalling pathways and natural killer cell-mediated cytotoxicity pathways were enriched during high GIMAP7 expression, indicating that GIMAP7 could modulate these pathways (Figure 6A). Conversely, pathways such as oxidative phosphorylation, glyoxylate and dicarboxylic acid metabolism and glycosylphospholipid anchor biosynthesis were enriched during low GIMAP7 expression, suggesting the inhibitory role of GIMAP7 in these pathways (Figure 6B). HALLMARK revealed that the pathways of allograft rejection, complement and IL2 

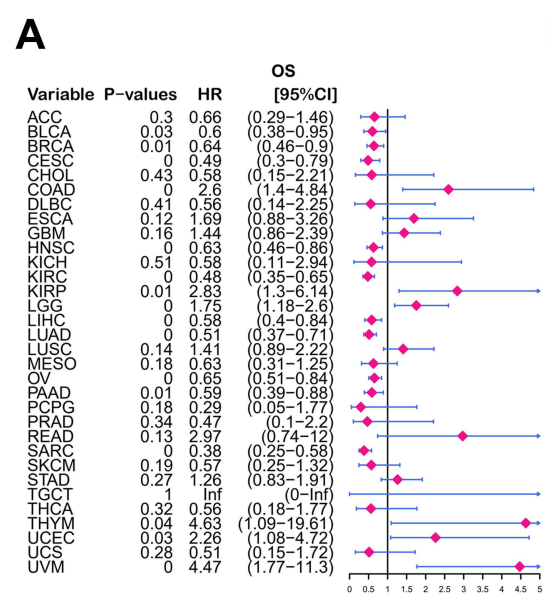

B

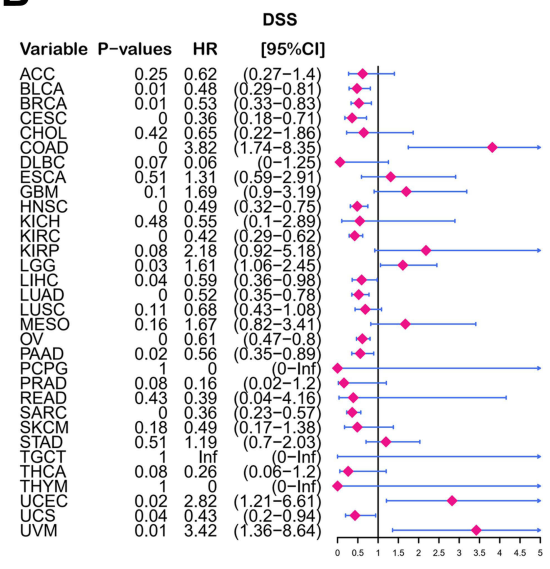

C

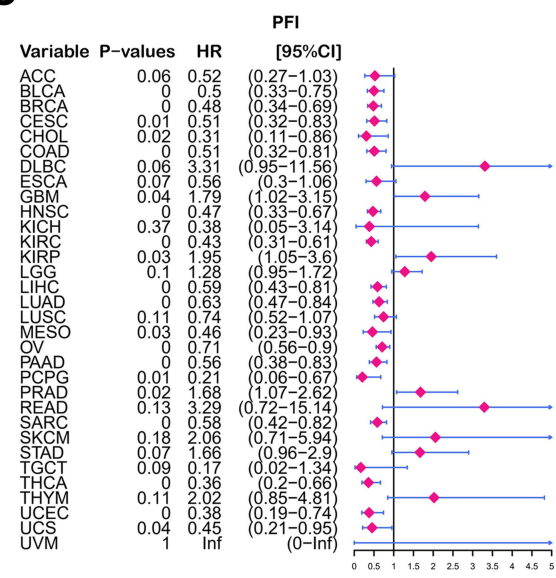

Figure 5 Prognostic analysis of GIMAP7 via multivariable Cox regression. (A) The results of GIMAP7 for overall survival in pan-cancer. (B) The results of GIMAP7 for disease-specific survival in pan-cancer. (C) The results of GIMAP7 for the progression-free interval in pan-cancer.

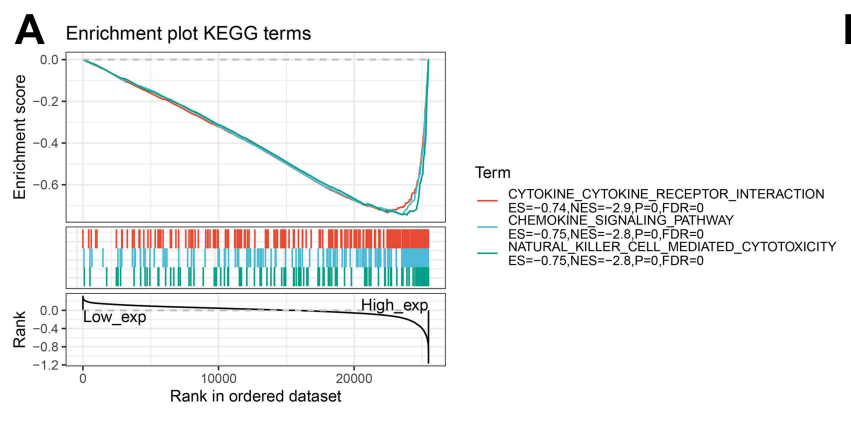

C Enrichment plot HALLMARK terms

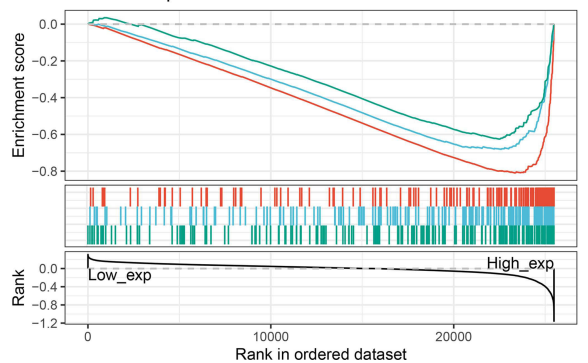

B Enrichment plot KEGG terms

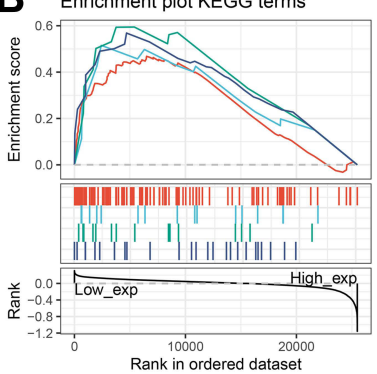

Term

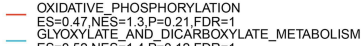

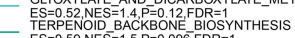

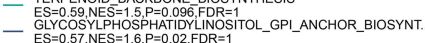

D Enrichment plot HALLMARK terms

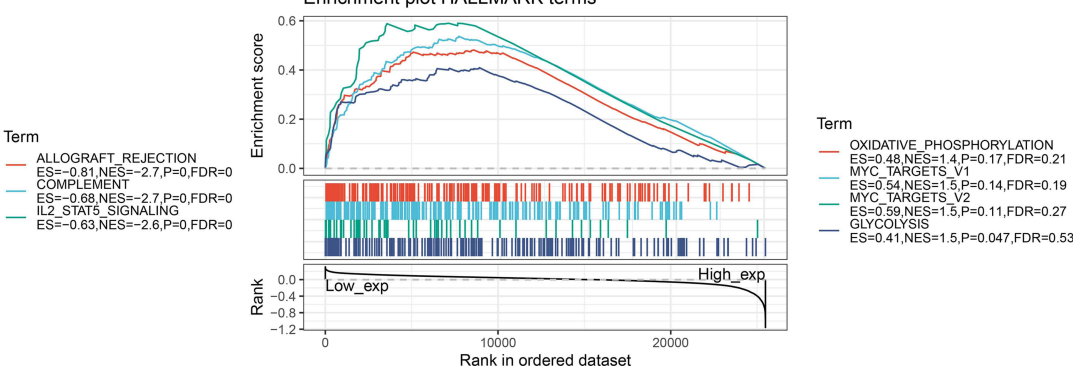

Figure 6 Gene set enrichment analysis of GIMAP7. (A and B) Functional terms linked with GIMAP7 enrichment via Kyoto Encyclopedia of Genes and Genomes (KEGG) pathway analysis. (C and D) Functional terms linked with GIMAP7 enrichment via HALLMARK pathway analysis.

STAT6 signalling were enriched during high GIMAP7 expression, which suggested that GIMAP7 may be positively related to these pathways (Figure 6C). Contrastingly, pathways such as oxidative phosphorylation, MYC target V1, MYC target V2 and glycolysis were enriched during low GIMAP7 expression, which suggested that GIMAP7 may be negatively related to these pathways (Figure 6D).

\section{Correlation Between the Immune Infiltration of CD8+ and CD4+ T Cells and GIMAP7}

Correlating the abundance of the immune cells, CD8 $+\mathrm{T}$ cells and CD4+ T cells, and GIMAP7 in different tumour types revealed that GIMAP7 positively correlated with the infiltration of CD8+ and CD4+ T cells in most of the tumours (Figure 7A and B). To understand the relationship between immune cells and GIMAP7 in various tumours, the abundance of 22 immune cells was calculated, wherein GIMAP7 was found to be significantly and positively correlated 
with CD8+ T cells, M1-like and M2-like macrophages. Additionally, GIMAP7 was negatively correlated with naive CD4 $+\mathrm{T}$ cells, activated myeloid dendritic cells and eosinophil $(\mathrm{P}<0.01$, Figure $7 \mathrm{C})$.

\section{Correlation Between GIMAP7 Expression and Tumour Purity, Immunoregulation Related Genes and Chemokines}

Purity of tumour was measured via estimation score, which is a combined ratio to assess the immune and stromal components in tumour tissue. GIMAP7 expression was specifically correlated with the estimate score in many cancers, such as CESC $(\mathrm{R}=0.804)$, LUSC $(\mathrm{R}=0.833)$, OV $(\mathrm{R}=0.813)$, SKCM $(\mathrm{R}=0.812)$ and STAD $(\mathrm{R}=0.822)$ (Supplementary Figure 5). The correlation between the stromal score and GIMAP7 was high in KICH $(\mathrm{R}=0.724)$, STAD $(\mathrm{R}=0.732), \operatorname{COAD}(\mathrm{R}=0.699)$, UCS $(\mathrm{R}=0.692)$ and prostate adenocarcinoma $(\mathrm{PRAD})(\mathrm{R}=0.69)$ (Supplementary Figure 6). Additionally, GIMAP7 was found to have a higher association with the immune score in CESC $(\mathrm{R}=0.791)$, HNSC $(\mathrm{R}=0.803)$, LUSC $(\mathrm{R}=0.883), \mathrm{OV}(\mathrm{R}=0.817), \mathrm{SKCM}(\mathrm{R}=0.814)$ and TGCT $(\mathrm{R}=0.862)$ than other cancer types (Supplementary Figure 7). These results suggest that high GIMAP7 expression is often accompanied by immune cell infiltration in the tumour microenvironment.

Moreover, GIMAP7 expression was found to be positively correlated with CD48, CD40LG and CD28 and negatively correlated with CD276 andTNFSF9. The negative correlation between GIMAP7 and CD276 was most evident in BRCA, LUAD, LUSC and THCA. Additionally, GIMAP7 was more significantly associated with ULBP1 in STAD and TGCT than TNFSF9 in LUAD ( $<$ 0.01) (Figure 8A). Immune inhibitors play a vital role in the immune regulation of tumours. This study suggests that GIMAP7 expression is significantly positively linked to TIGIT and CD96 in most tumours but significantly negatively linked to PVRL2 in PRAD $(\mathrm{P}<0.001)$ (Figure $8 \mathrm{~B})$.

Further investigation of the specific association between GIMAP7 expression in pan-cancer and chemokines and their receptors revealed that CCL5 and XCL2, which are chemokines, were positively and prominently associated with GIMAP7 expression in most cancers. Moreover, chemokine receptors, such as CCR2, CXR1 and CCR5, were positively and significantly related to GIMAP7 expression $(\mathrm{P}<0.01)$ (Figure $8 \mathrm{C}$ and $\mathrm{D}$ ).

\section{Relationship Between GIMAP7 and Immune Checkpoint Genes in Pan-Cancer}

Immune checkpoints are important targets for immunotherapy. ${ }^{15}$ This study unravels the relationship between more than 40 immune checkpoint genes and GIMAP7 expression. GIMAP7 was significantly and positively correlated with the expression levels of most immune checkpoints. Interestingly, GIMAP7 was significantly correlated with the expression levels of PD-L1 and PD1 in over two-thirds of the tumour types analysed $(\mathrm{P}<0.05)$. GIMAP7 expressed in UVM showed the most significant positive association with 39 out of 47 immune checkpoints $(\mathrm{P}<0.001)$, while it was expressed in 35 out of 47 in PRAD $(\mathrm{P}<0.01)$. However, there was no statistically significant correlation between GIMAP7 expression and the immune checkpoint genes in PCPG (Supplementary Figure 8).

\section{Relationship of GIMAP7 Expression with TMB and MSI}

TMB and MSI are considered important factors that reflect prognosis and immune response. Previous studies have reported that high TMB levels are associated with high MSI levels. ${ }^{16}$ Thus, the relationship between MSI and TMB with GIMAP7 expression could be a possible guide for clinical immunotherapy for tumours with differential GIMAP7 expression. The positive correlation between GIMAP7 expression and high TMB was significant in UVM $(\mathrm{P}=0.007)$, UCS ( $\mathrm{P}=0.029)$, UCEC $(\mathrm{p}=0.037)$, OV $(\mathrm{P}=0.0013)$, LGG $(\mathrm{P}=0.0023)$ and COAD $(\mathrm{P}=0.025)$ ( Supplementary Figure 9A). Likewise, the positive correlation between GIMAP7 expression and MSI was significant in READ $(\mathrm{P}=$ $0.0011)$ and COAD $(\mathrm{P}=2.7 \mathrm{e}-08)$ (Supplementary Figure 9B).

\section{Association Between GIMAP7 Expression and ICB Response}

The correlation between GIMAP7 expression and ICB response was validated by analysing data from the immunotherapy cohort of uroepithelial carcinoma (IMvigor210). Significant differences were found in GIMAP7 expression in patients with different efficacies. GIMAP7 expression was highest in patients with complete response, lowest in patients 
A

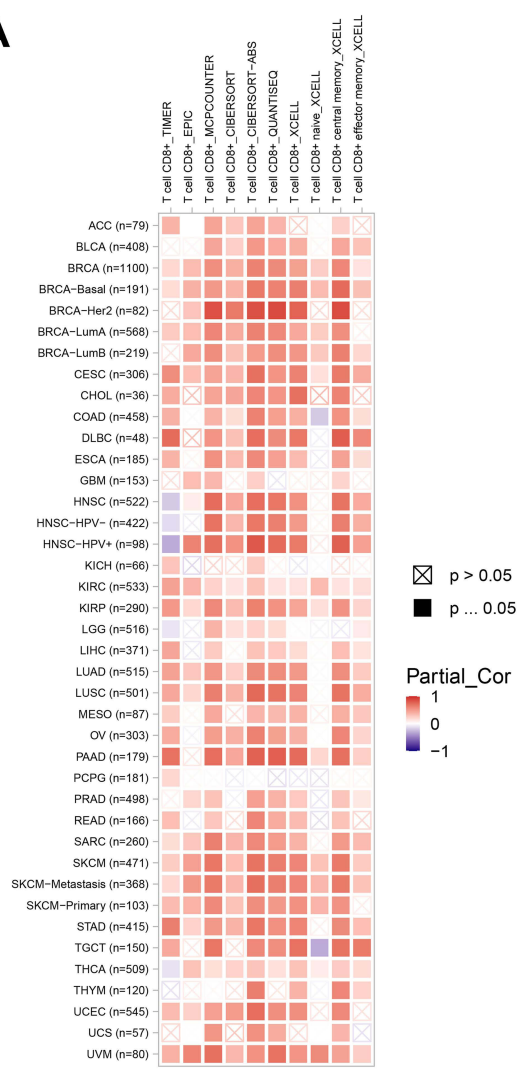

B

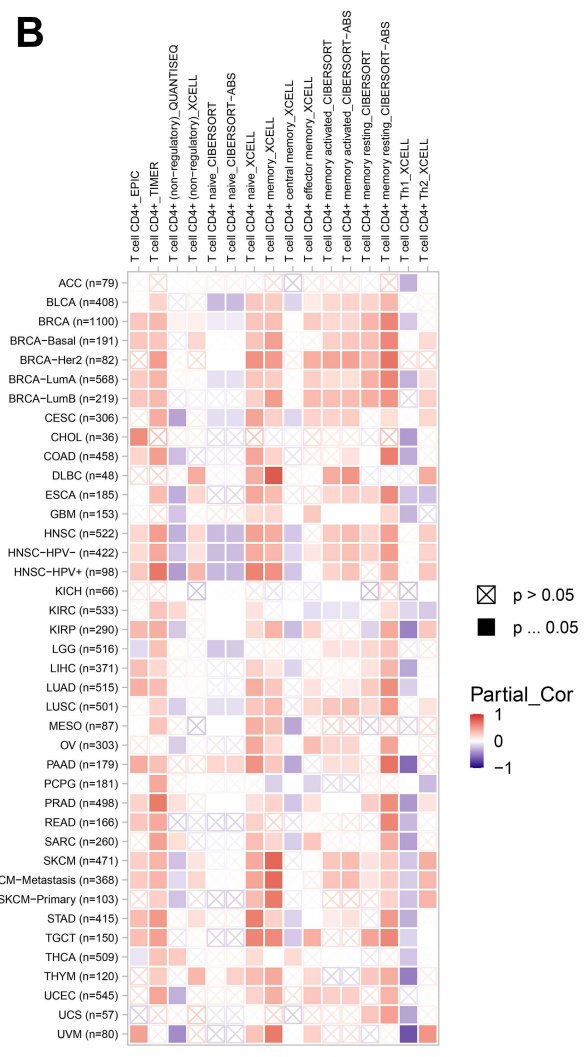

C

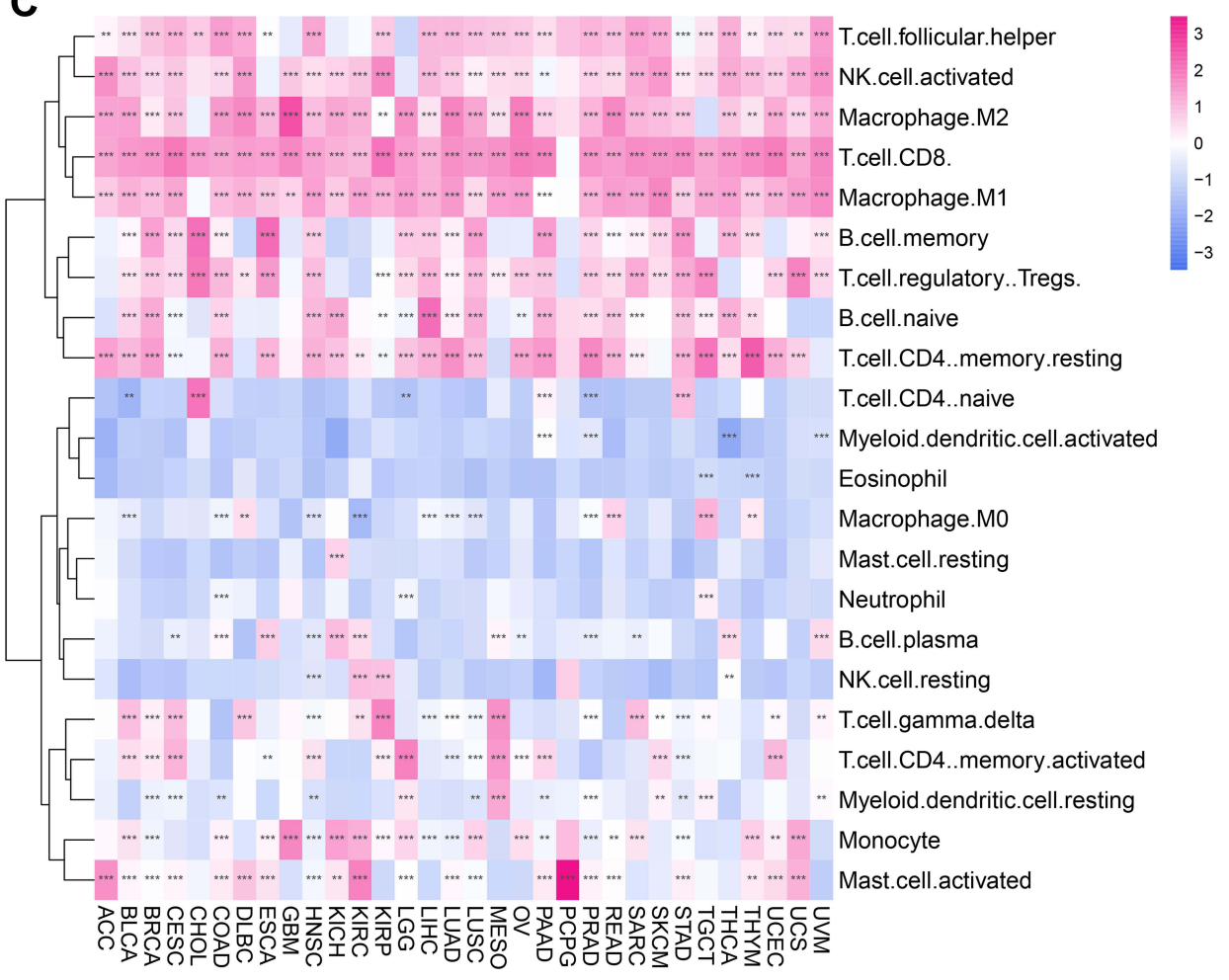

Figure 7 Analysis of the correlation between GIMAP7 expression and immune cell infiltration. (A) The correlation between GIMAP7 expression and CD8+ T cell infiltration level in pan-cancer. (B) The correlation between GIMAP7 expression and CD4+ T cell infiltration level in pan-cancer. (C) The correlation between GIMAP7 expression and different immune cell infiltrations in pan-cancer. $* \mathrm{P}<0.05$, $* * \mathrm{P}<0.01, * * * \mathrm{P}<0.001$ 
A

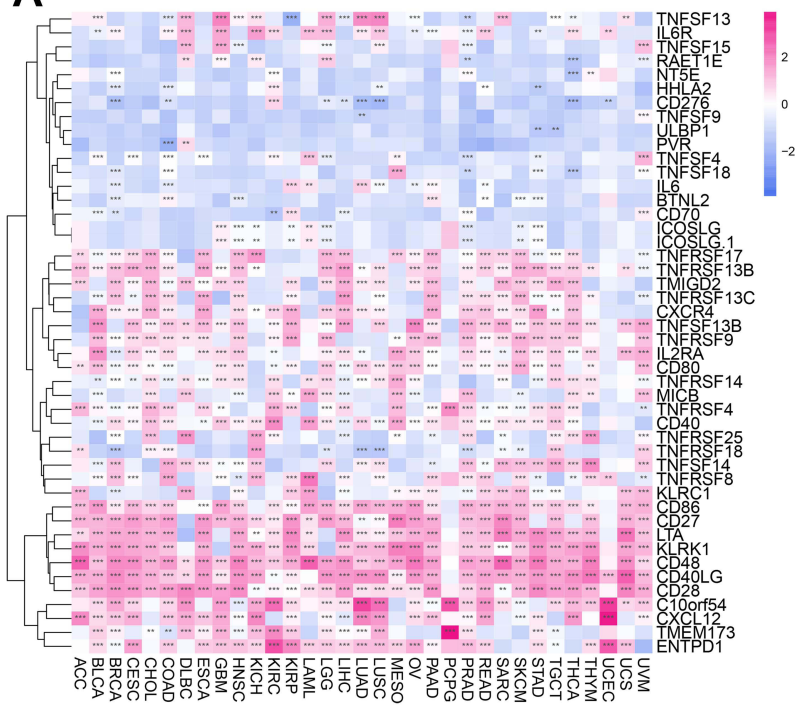

C

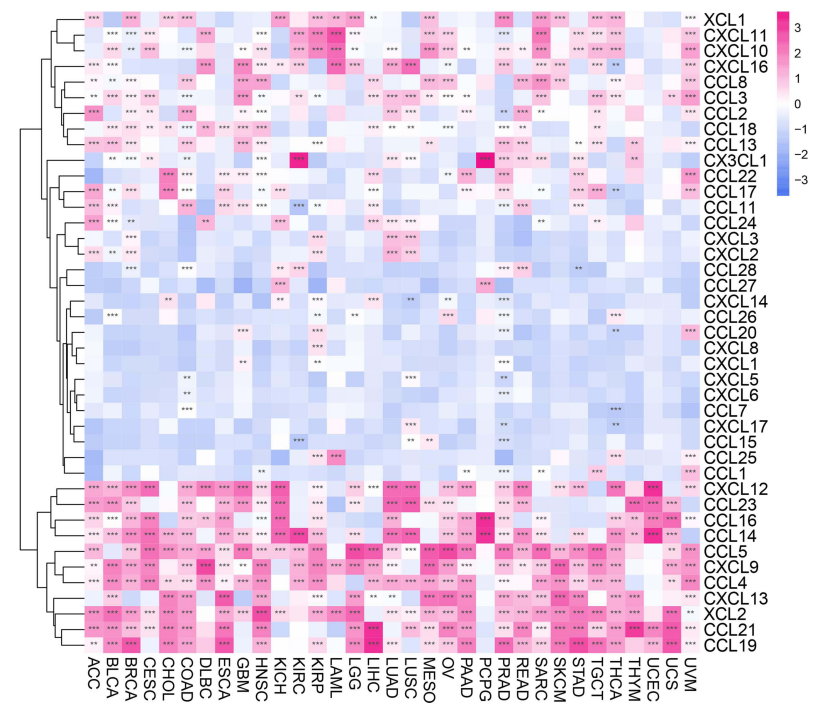

B

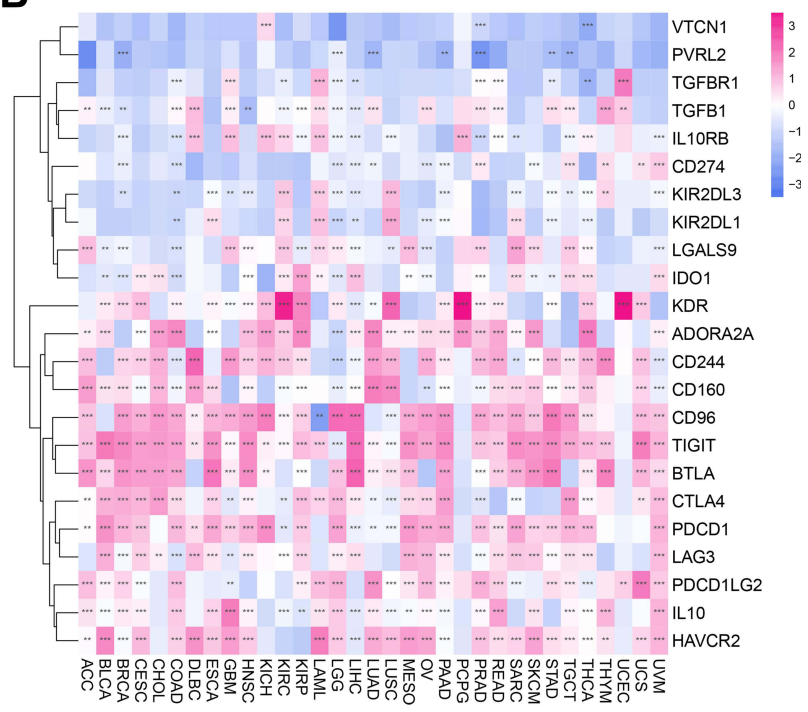

D

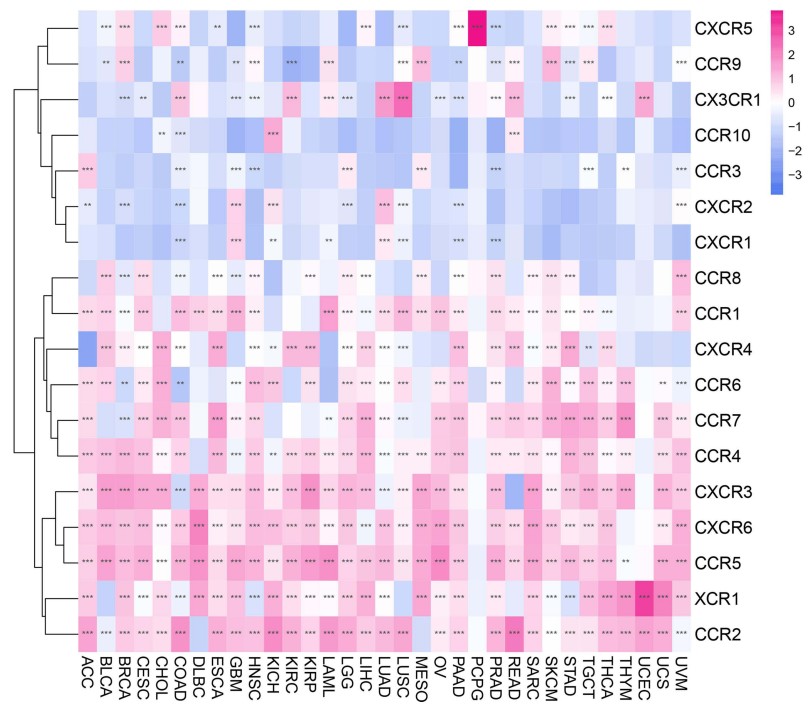

Figure 8 Association between GIMAP7 and immunoregulation-related molecules. (A) The relationship between GIMAP7 expression and immune-activating genes. (B) The relationship between GIMAP7 expression and immunosuppressive status-related genes. (C) The relationship between GIMAP7 expression and chemokine genes. (D) The relationship between GIMAP7 expression and chemokine receptor genes. ${ }^{*} \mathrm{P}<0.01$; $* * * \mathrm{P}<0.00 \mathrm{I}$.

with progressive disease and intermediate in patients with stable disease $(\mathrm{P}=0.048$, Figure 9A). This suggests that patients with high GIMAP7 expression could be a potentially beneficial population for ICB treatment. TIDE score is currently the most promising marker of ICB response and has been reported to have higher accuracy than PD-L1 expression level and TMB in predicting the survival outcome of patients with cancer who are treated with ICB agents. ${ }^{14,17,18}$ Patients with high TIDE scores have a higher chance of tumour immune escape, and thus exhibit a lower response rate of ICB treatment. A significant negative correlation between GIMAP7 expression and TIDE score was observed in all the tumours analysed (Figure 9B; Supplementary Figure 10). These results suggest that GIMAP7 expression is associated with ICB response and could be a potential marker for ICB treatment. 
A

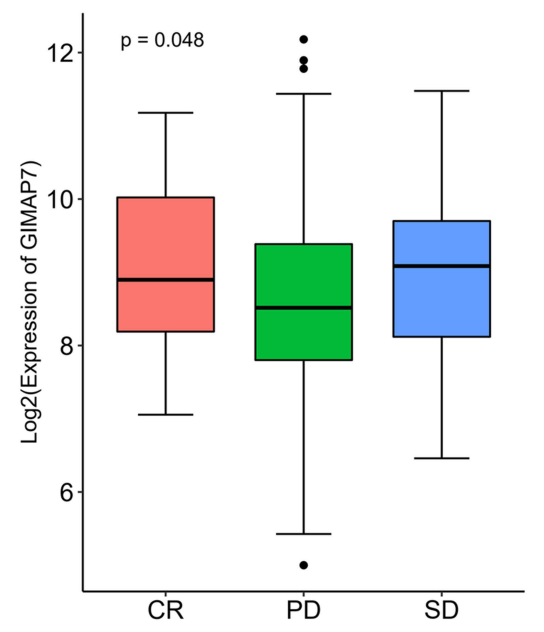

B

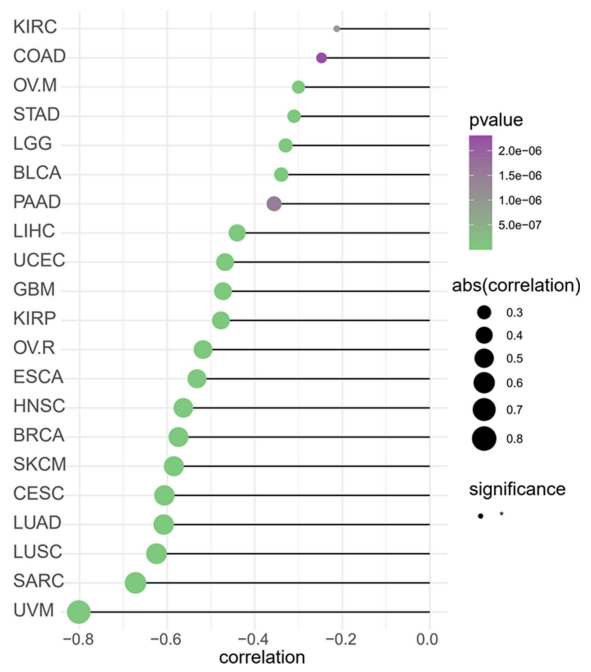

Figure 9 The association of GIMAP7 expression with immune checkpoint blockade (ICB) response and Tumour Immune Dysfunction and Exclusion (TIDE) scores. (A) The association between GIMAP7 expression and ICB response. Data from the immunotherapy cohort IMvigor2I0, showing complete response (CR), progressive disease (PD) and stable disease (SD). (B) The association between GIMAP7 expression and TIDE score. The larger the point the larger the absolute correlation coefficient value; the colours indicate $P$ values, whereas dots indicate $P<0.05$.

\section{Discussion}

The expression, mutations, copy number variation, prognostic value, immune infiltration relationship and association with GIMAP7 immunotherapeutic markers at pan-cancer levels have been investigated in this study. GIMAP7 was found to be significantly underexpressed in most tumours, with low expression associated with the advanced tumour stage. GIMAP7 was considered an independent prognostic factor in many tumours, and patients with high GIMAP7 expression had a better prognosis than those with low GIMAP7 expression. GIMAP7 correlated with CD8+ and CD4+ T cell abundance in the tumour microenvironment and with various immune checkpoint molecules. Analysis of immunotherapy cohort data suggests that GIMAP7 expression level is closely related to the patient's response to immunotherapy. Therefore, GIMAP7 could be a potential prognostic and predictive marker for immunotherapy.

The evaluation of mRNA expression of GIMAP7 in human tumours showed that GIMAP7 had significantly lower expression in most cancers. GIMAP7 has been demonstrated to be a central gene in head and neck cancers with low expression, ${ }^{19}$ which is concordant with our findings. However, differences between the results of the Oncomine and TCGA databases were observed, which could be attributed to the fact that the Oncomine database is based on the secondary analysis of data uploaded to the GEO database by individual researchers worldwide. The inconsistent inclusion and exclusion criteria and inconsistent data standardisation methods between studies have contributed to the high variability between the data.

Furthermore, GIMAP7 was found to be closely related to various immune pathways, including cytokine receptor interactions, which provide potential markers for tumour therapeutics. Chemokines are cytokines that induce directional cell movement, induce an immune response and participate in immune regulation and related pathological responses. ${ }^{20}$ They are also associated with tumour development. ${ }^{21}$ Leukocytes are dependent on the activation of chemokine integrins, which allows them to adhere to the luminal surface of blood vessels and migrate further outside the blood vessels to the site of inflammation. ${ }^{22}$ However, the large number of leukocytes recruited by chemokines can have damaging effects, and the continued high expression (or increased expression) of chemokines due to positive physiological feedback mechanisms can further cause extensive tissue damage. This ultimately leads to the destruction of the protective effect of leukocytes on the body, organismal pathology and an increased probability of cancer. ${ }^{23,24}$ Chemokines have a two-fold mechanism: promoting angiogenesis and tumour proliferation; inhibiting angiogenesis and tumour cell growth. Some chemokines have promising applications in the physiological treatment of malignant tumours due to their ability to attract immune effector cells and inhibit angiogenesis, thus combating tumour growth and metastasis. Moreover, some 
chemokine receptors have the potential to be drug targets in various diseases. ${ }^{25,26}$ GIMAP7 is associated with the cytokine receptor interaction pathway, indicating its role in the induction of immune cell recruitment. Therefore, the intervention of GIMAP7 expression could impact the tumour immune microenvironment.

Consistent with previous studies, GIMAP7 was significantly and positively correlated with CD8+ and CD4+ T cells. Moreover, a previous study has argued that GIMAPs are involved in the functioning of the immune system, particularly $\mathrm{T}$ cells that support and control the immune process. ${ }^{27}$ The expression of GIMAP7 has been reported to increase during the conversion from $\mathrm{CD} 4+\mathrm{CD} 8+$ double-positive cells to $\mathrm{CD} 4+$ or $\mathrm{CD} 8+$ single-positive cells in the mouse thymus. ${ }^{28}$ Nitta and Takahama report that the GIMAP proteins play a centric role in T cell growth and development. ${ }^{29}$ Therefore, GIMAP7 could be hypothesised to have regulatory effects on T cells in the tumour microenvironment; however, further studies are required.

Finally, the potential of GIMAP7 as a therapeutic marker for ICB was evaluated. PD-L1 expression, TMB, MSI and TIDE score are currently the recognised markers of ICB therapy. GIMAP7 was found to positively correlate with PD-L1 expression levels in most tumours, suggesting that high GIMAP7 expression could be associated with better ICB efficacy. Similarly in triple-negative breast cancer, GIMAP7 was identified as a core immune-related gene and positively correlated with PD-L1 expression. ${ }^{30} \mathrm{TMB}$ refers to the number of somatic mutations in the tumour genome after germline mutations have been removed. The higher the TMB; the greater the number of neoantigens expressed by the tumour cell, which are more likely to be recognised by $\mathrm{T}$ cells and induce the body's anti-tumour immune response. $^{31,32}$ MSI, which is a clinically important tumour marker, occurs because of a functional defect in DNA mismatch repair in tumour tissues. ${ }^{33}$ In 2017, the FDA approved the PD-1 inhibitor Keytruda for the treatment of patients with any solid tumour with MSI-H. This is the first immunotherapy that does not differentiate based on the tumour origin, but rather differentiates based on tumour markers. ${ }^{34}$ TIDE score is currently the most promising marker of ICB response, with various studies suggesting a higher accuracy of TIDE score than PD-L1 expression level and TMB in predicting the survival outcome of patients with cancer who are treated with ICB agents. Our results show that GIMAP7 is significantly associated with these three markers, indicating the potential of GIMAP7 as an ICB marker. Upon investigation of the correlation between GIMAP7 and ICB response using a real ICB treatment cohort, GIMAP7 expression was found to be lowest in patients with progressive diseases but highest in patients in complete remission.

Despite the systematic analysis of GIMAP7, there are inevitable limitations in this study. First, the exact significance of GIMAP7 expression in various cancers and the exact implication of immunoregulation in these cancers remain incomplete. Second, direct clinical trials are further required to clarify the capacity of GIMAP7 in regulating the immune process.

\section{Conclusions}

The systematic analysis of GIMAP7 in pan-cancer has shown that GIMAP7 is a protective factor in most cancers and is a promising anti-cancer gene, mainly involved in anti-tumour immune pathways. This study lays the foundation for subsequent functional and mechanistic experiments, which may have clinical implications.

\section{Data Sharing Statement}

The relevant datasets are included in the article.

\section{Ethics Approval and Consent to Participate}

This study was approved by the Ethics and Human Subject Committee of Guangxi Medical University Cancer Hospital.

\section{Author Contributions}

All authors made substantial contributions to conception and design, the acquisition of data, or analysis and interpretation of data; took part in drafting the article or revising it critically for important intellectual content; agreed to the current journal submission; approved the version to be published; and agreed to be accountable for all aspects of the work. 


\section{Funding}

This work was funded by the Guangxi Multidisciplinary Collaborative Health Management Talent Mini-Highland (guizutongzi:2019-85).

\section{Disclosure}

The authors report no conflicts of interest relevant to this work.

\section{References}

1. Bray F, Ferlay J, Soerjomataram I, Siegel RL, Torre LA, Jemal A. Global cancer statistics 2018: GLOBOCAN estimates of incidence and mortality worldwide for 36 cancers in 185 countries. CA Cancer J Clin. 2018;68(6):394-424. doi:10.3322/caac.21492

2. Bindea G, Mlecnik B, Tosolini M, et al. Spatiotemporal dynamics of intratumoral immune cells reveal the immune landscape in human cancer. Immunity. 2013;39(4):782-795. doi:10.1016/j.immuni.2013.10.003

3. Zhang W, Xu S, Wu G, Liu Y, Wang Q, Man C. Exploring the expression and preliminary function of chicken Gimap5 gene. PeerJ. $2019 ; 7$ :e7618. doi: $10.7717 /$ peerj.7618

4. Krucken J, Schroetel RM, Muller IU, et al. Comparative analysis of the human gimap gene cluster encoding a novel GTPase family. Gene. 2004;341:291-304. doi:10.1016/j.gene.2004.07.005

5. Limoges MA, Cloutier M, Nandi M, Ilangumaran S, Ramanathan S. The GIMAP family proteins: an incomplete puzzle. Front Immunol. 2021;12:679739. doi:10.3389/fimmu.2021.679739

6. Deng S, Zhang Z, Lu X, Zhou Q, Xia S, Li M. Systemic analyses of expression patterns and clinical features for GIMAPs family members in lung adenocarcinoma. Aging. 2020;12(20):20413-20431. doi:10.18632/aging.103836

7. Webb LM, Datta P, Bell SE, Kitamura D, Turner M, Butcher GW. GIMAP1 is essential for the survival of naive and activated B cells in vivo. J Immunol. 2016;196(1):207-216. doi:10.4049/jimmunol.1501582

8. Schnell S, Démollière C, van den Berk P, Jacobs H. Gimap4 accelerates T-cell death. Blood. 2006;108(2):591-599. doi:10.1182/blood-2005-114616

9. Schwefel D, Arasu BS, Marino SF, et al. Structural insights into the mechanism of GTPase activation in the GIMAP family. Structure. 2013;21 (4):550-559. doi:10.1016/j.str.2013.01.014

10. Goldman MJ, Craft B, Hastie M, et al. Visualizing and interpreting cancer genomics data via the Xena platform. Nat Biotechnol. 2020;38 (6):675-678. doi:10.1038/s41587-020-0546-8

11. Hoadley KA, Yau C, Hinoue T, et al. Cell-of-origin patterns dominate the molecular classification of 10,000 tumors from 33 types of cancer. Cell. 2018;173(2):291-304.e296. doi:10.1016/j.cell.2018.03.022

12. Mariathasan S, Turley SJ, Nickles D, et al. TGFbeta attenuates tumour response to PD-L1 blockade by contributing to exclusion of T cells. Nature. 2018;554(7693):544-548. doi:10.1038/nature25501

13. Lan H, Yuan J, Chen X, et al. Multiomics profiling of the expression and prognosis of MCMs in endometrial carcinoma. Biosci Rep. 2021. doi:10.1042/BSR20211719

14. Jiang P, Gu S, Pan D, et al. Signatures of T cell dysfunction and exclusion predict cancer immunotherapy response. Nat Med. 2018;24 (10):1550-1558. doi:10.1038/s41591-018-0136-1

15. Tooyserkani R, Rasaee MJ, Bandehpour M, WPM Löwik D. Novel anti-PD-L1 peptide selected from combinatorial phage library inhibits tumor cell growth and restores T-cell activity. J Drug Target. 2021;29(7):771-782. doi:10.1080/1061186X.2021.1879087

16. Goodman AM, Sokol ES, Frampton GM, Lippman SM, Kurzrock R. Microsatellite-stable tumors with high mutational burden benefit from immunotherapy. Cancer Immunol Res. 2019;7(10):1570-1573. doi:10.1158/2326-6066.CIR-19-0149

17. Wang S, He Z, Wang X, Li H, Liu XS. Antigen presentation and tumor immunogenicity in cancer immunotherapy response prediction. eLife. 2019;8:e49020

18. Kaderbhaï C, Tharin Z, Ghiringhelli F. The role of molecular profiling to predict the response to immune checkpoint inhibitors in lung cancer. Cancers. 2019;11(2). doi:10.3390/cancers 11020201

19. Song Y, Pan Y, Liu J. The relevance between the immune response-related gene module and clinical traits in head and neck squamous cell carcinoma. Cancer Manag Res. 2019;11:7455-7472. doi:10.2147/CMAR.S201177

20. Mo X, Huang X, Feng Y, et al. Immune infiltration and immune gene signature predict the response to fluoropyrimidine-based chemotherapy in colorectal cancer patients. Oncoimmunology. 2020;9(1):1832347. doi:10.1080/2162402X.2020.1832347

21. Yang M, Li J, Gu P, Fan X. The application of nanoparticles in cancer immunotherapy: targeting tumor microenvironment. Bioact Mater. 2021;6 (7):1973-1987. doi:10.1016/j.bioactmat.2020.12.010

22. Wu G, Liu J, Liu H, et al. An applicable inflammation-joined and nutrition-related prognostic indicator in patients with colorectal cancer. Front Oncol. 2021;11(2332):644670.

23. Chen L, Deng H, Cui H, et al. Inflammatory responses and inflammation-associated diseases in organs. Oncotarget. 2018;9(6):7204-7218. doi:10.18632/oncotarget.23208

24. Hughes CE, Nibbs RJB. A guide to chemokines and their receptors. FEBS J. 2018;285(16):2944-2971. doi:10.1111/febs.14466

25. Yue Y, Lian J, Wang T, et al. Interleukin-33-nuclear factor-KB-CCL2 signaling pathway promotes progression of esophageal squamous cell carcinoma by directing regulatory T cells. Cancer Sci. 2020;111(3):795-806. doi:10.1111/cas.14293

26. Liu J, Huang X, Liu H, et al. Immune landscape and prognostic immune-related genes in KRAS-mutant colorectal cancer patients. $J$ Transl Med. 2021;19(1):27. doi:10.1186/s12967-020-02638-9

27. Ciucci T, Bosselut R. Gimap and T cells: a matter of life or death. Eur J Immunol. 2014;44(2):348-351. doi:10.1002/eji.201344375

28. Nitta T, Nasreen M, Seike T, et al. IAN family critically regulates survival and development of T lymphocytes. PLoS Biol. 2006;4(4):e103. doi:10.1371/journal.pbio.0040103 
29. Nitta T, Takahama Y. The lymphocyte guard-IANs: regulation of lymphocyte survival by IAN/GIMAP family proteins. Trends Immunol. 2007;28 (2):58-65. doi:10.1016/j.it.2006.12.002

30. Zhang J, Wang $\mathrm{L}, \mathrm{Xu} \mathrm{X}$, et al. Transcriptome-based network analysis unveils eight immune-related genes as molecular signatures in the immunomodulatory subtype of triple-negative breast cancer. Front Oncol. 2020;10:1787. doi:10.3389/fonc.2020.01787

31. Yarchoan M, Hopkins A, Jaffee EM. Tumor mutational burden and response rate to PD-1 inhibition. $N$ Engl J Med. 2017;377(25):2500-2501. doi:10.1056/NEJMc1713444

32. Huang J, Liu H, Zhao Y, et al. MicroRNAs expression patterns predict tumor mutational burden in colorectal cancer. Front Oncol. $2020 ; 10: 550986$. doi:10.3389/fonc. 2020.550986

33. Huang X, Liu J, Liu H, et al. A combined epithelial mesenchymal transformation and DNA repair gene panel in colorectal cancer with prognostic and therapeutic implication. Front Oncol. 2020;10:595182. doi:10.3389/fonc.2020.595182

34. Lemery S, Keegan P, Pazdur R. First FDA approval agnostic of cancer site - when a biomarker defines the indication. $N$ Engl J Med. 2017;377 (15):1409-1412. doi:10.1056/NEJMp1709968

Journal of Inflammation Research

\section{Publish your work in this journal}

The Journal of Inflammation Research is an international, peer-reviewed open-access journal that welcomes laboratory and clinical findings on the molecular basis, cell biology and pharmacology of inflammation including original research, reviews, symposium reports, hypothesis formation and commentaries on: acute/chronic inflammation; mediators of inflammation; cellular processes; molecular mechanisms; pharmacology and novel anti-inflammatory drugs; clinical conditions involving inflammation. The manuscript management system is completely online and includes a very quick and fair peer-review system. Visit http://www.dovepress.com/testimonials.php to read real quotes from published authors.

Submit your manuscript here: https://www.dovepress.com/journal-of-inflammation-research-journal 